Europhys. Lett., 53 (1), pp. 46-52 (2001)

\title{
Mean-field approach to polymeric microemulsions
}

\author{
H. Kodama ${ }^{1,2}$, S. Komura ${ }^{3}$ and K. TamurA ${ }^{3}$ \\ 1 Japan Chemical Innovation Institute, Nagoya University \\ Nagoya 464-8601, Japan \\ 2 Yokohama Research Center, Mitsubishi Chemical Corporation \\ Yokohama 227-8502, Japan \\ 3 Faculty of Computer Science and Systems Engineering, \\ Kyushu Institute of Technology - Iizuka 820-8502, Japan
}

(received 9 November 1999; accepted in final form 20 October 2000)

PACS. 64.75.+g - Solubility, segregation, and mixing; phase separation. PACS. 82.20.Wt - Computational modeling; simulation.

PACS. 82.70.Kj - Emulsions and suspensions.

\begin{abstract}
Equilibrium structure of ternary blends of $A$ and $B$ homopolymers and symmetric $A B$ block copolymer is investigated using an extended lattice self-consistent field theory by means of numerical calculations. We demonstrate that bicontinuous polymeric microemulsions result from microphase separation for relatively longer copolymer chain. Detailed free energy analysis revealed that the stability of the lamellar phase is marginal in the corresponding region. For relatively shorter copolymer chain, 3-phase coexistence between the lamellar, $A$-rich and $B$-rich phases is observed.
\end{abstract}

Introduction. - Blends of homopolymers and block copolymers have been stimulating a considerable amount of experimental and theoretical studies because of the richness in their phase behavior as well as their technological importance [1]. Such systems are known to form either mesoscopically structured phases with long-range order (LRO) or a homogeneous phase depending on the temperature or the composition. Recently, Bates et al. found a new bicontinuous phase without any LRO in a ternary blend of $A$ and $B$ homopolymers of equal sizes and a symmetric $A B$ diblock copolymer [2,3]. This phase has been termed as "polymeric microemulsion" (PME) and its physical origin is of great interest and importance to understand.

In the above experiment, the average volume fraction of the copolymer (denoted by $\theta$ ) is changed, while the volume fractions of the two homopolymers are kept to be equal. This system exhibits both macrophase and microphase separations into 2-phase and lamellar 1phase for small and large $\theta$, respectively. PME has been observed for intermediate $\theta$ but within a narrow region close to the mean-field Lifshitz volume fraction $\theta_{\mathrm{L}}[4-6]$. Bates et al. assumed that, due to the strong fluctuations, a singular cusp in the transition temperature is induced around $\theta_{\mathrm{L}}$, and PME is regarded as a strongly fluctuating disordered phase $[2,3]$.

From the theoretical point of view, the appearance of PME in the polymeric ternary system has not yet been well understood, although various structures with LRO have been successfully explained within the self-consistent field theory (SCFT) [7-9]. In these works, the free energies of several periodic structures were calculated by assuming their mesophase symmetry a priori. Hence this approach is not suitable for dealing with structures without any LRO such as PME. On the other hand, there is another approach in which the SCFT (c) EDP Sciences 
equations are solved numerically without assuming the symmetry of the structure $[10,11]$. Although this method generates the structures corresponding to either stable or metastable equilibrium, it has proved to be a powerful tool in finding new mesophases.

In this letter, using the extended lattice SCFT method with randomly generated initial potential fields, we demonstrate that the bicontinuous structure of PME results from microphase separation for relatively longer copolymer chain. However, since the obtained PME may correspond to a metastable equilibrium, we have also performed the same numerical calculation starting from the initial potential fields with an imposed lamellar symmetry. By examining the free energy of the lamellar phase as a function of $\theta$, we find that there is a region where the stability of the lamellar phase is marginal. This means that the copolymer monolayers do not interact each other in the corresponding region.

Lattice SCFT and simulation method. - We first describe the lattice SCFT for general copolymer mixtures [12]. The space coordinate of each segment composing the copolymers is specified by a lattice point $\boldsymbol{n}=\left(n_{x}, n_{y}, n_{z}\right)$ in an $L_{x} \times L_{y} \times L_{z}$ cubic lattice box with a lattice constant $b$ with periodic boundary conditions. We denote the type of molecule by $i$, and the type of segment by $J$ and $K$. Segments in a chain are indexed by $N^{\prime}=1, \cdots, N_{i}$, where $N_{i}$ is the polymerization index of the $i$-th molecule. The molecules of type $i$ are indexed by $a=1, \cdots, n_{i}$, where $n_{i}$ is the total number of the $i$-th molecule. The configuration space is denoted by $\Gamma \equiv\left\{\boldsymbol{n}_{a, N^{\prime}}^{(i)}\right\}$. In order to explain how we extended the lattice SCFT, we start with the Hamiltonian description. Throughout this letter, all the energies are expressed in units of $k_{\mathrm{B}} v T$, where $k_{\mathrm{B}}$ and $T$ represent the Boltzmann constant and the temperature, respectively.

The Hamiltonian of the system is composed of two parts:

$$
\hat{\mathcal{H}}(\Gamma)=\hat{\mathcal{H}}_{0}(\Gamma)+\hat{\mathcal{W}}(\Gamma)
$$

where the first and the second terms represent the ideal and the interaction Hamiltonians, respectively. The ideal Hamiltonian $\hat{\mathcal{H}}_{0}$ describes independent polymer chains represented as random walks, and is given by

$$
\exp \left[-\hat{\mathcal{H}}_{0}(\Gamma)\right]=\prod_{i, a, N^{\prime}} \delta\left[\left|\boldsymbol{n}_{a, N^{\prime}+1}^{(i)}-\boldsymbol{n}_{a, N^{\prime}}^{(i)}\right|, 1\right]
$$

where $\delta[$,$] is a Kronecker delta-function. The interaction Hamiltonian \hat{\mathcal{W}}(\Gamma)$ is assumed to have the form of $\hat{\mathcal{W}}(\Gamma)=\mathcal{W}\left[\left\{\hat{\phi}_{J}(\boldsymbol{n} ; \Gamma)\right\}\right]$. Here $\hat{\phi}_{J}(\boldsymbol{n} ; \Gamma)=\sum_{i, a, N^{\prime}} \delta\left[\boldsymbol{n}, \boldsymbol{n}_{a, N^{\prime}}^{(i)}\right] \delta\left[J, f_{i}\left(N^{\prime}\right)\right]$ is the microscopic representation of the segment density field, where $f_{i}\left(N^{\prime}\right)$ is a "type function" representing the type of the segment $N^{\prime}$ in the chain $i$. The functional form of $\mathcal{W}$ is given by

$$
\mathcal{W}\left[\left\{\phi_{J}(\boldsymbol{n})\right\}\right]=\frac{1}{2} \sum_{\boldsymbol{n}} \sum_{J, K} \chi_{J K} \phi_{J}(\boldsymbol{n})\left\langle\left\langle\phi_{K}(\boldsymbol{n})\right\rangle\right\rangle,
$$

where $\chi_{J K}$ is the Flory-Huggins interaction parameter and $\langle\langle X(\boldsymbol{n})\rangle\rangle$ denotes the average of $X(\boldsymbol{n})$ over the nearest-neighbor lattice sites, i.e., $\langle\langle X(\boldsymbol{n})\rangle\rangle=(1 / 6) \sum_{\boldsymbol{n}^{\prime}} \delta\left[\left|\boldsymbol{n}-\boldsymbol{n}^{\prime}\right|, 1\right] X\left(\boldsymbol{n}^{\prime}\right)$. The partition function is calculated by $\mathcal{Z}=\left(1 / \prod_{i} n_{i} !\right) \sum_{\Gamma} \exp [-\hat{\mathcal{H}}(\Gamma)]$. As a reference state to compare the energy, we choose a pure system in which the segment interactions are switched off. Hence the partition function of this reference system is given by $\mathcal{Z}_{0}=$ $\prod_{i}\left(n_{i} N_{i} z^{N_{i}-1}\right)^{n_{i}} / n_{i}$ !, where $z$ is the coordination number.

Using the mean-field approximation, we can express the free energy relative to this reference state as a functional of the segment density $\left\{\phi_{J}(\boldsymbol{n})\right\}$ and the segmental potential fields 
$\left\{U_{J}(\boldsymbol{n})\right\}$ conjugate to $\left\{\phi_{J}(\boldsymbol{n})\right\}$,

$$
F=-\log \left(\frac{\mathcal{Z}}{\mathcal{Z}_{0}}\right) \approx-\sum_{i} n_{i} \log \frac{\sum_{\boldsymbol{n}} G_{i}\left(\boldsymbol{n}, N_{i}\right)}{n_{i} N_{i}}+\mathcal{W}\left[\left\{\phi_{J}(\boldsymbol{n})\right\}\right]-\sum_{J} \sum_{\boldsymbol{n}} U_{J}(\boldsymbol{n}) \phi_{J}(\boldsymbol{n}),
$$

which should be minimized under a suitable constraint. In the above, we have introduced the single-chain statistical weight $G_{i}(\boldsymbol{n}, N)$ for a chain $i$ under the segment potential $\left\{U_{J}(\boldsymbol{n})\right\}$ starting from the free segment $N^{\prime}=1$ and having the segment $N^{\prime}=N$ at $\boldsymbol{n}$. Similarly, the conjugate weight $G_{i}^{\dagger}(\boldsymbol{n}, N)$ is defined for a chain having the free segment $N^{\prime}=N_{i}$. Minimizing $F$ with respect to both $\left\{\phi_{J}(\boldsymbol{n})\right\}$ and $\left\{U_{J}(\boldsymbol{n})\right\}$ subjected to the constraint of local incompressibility, $\sum_{J} \phi_{J}(\boldsymbol{n})=1$, we obtain the set of SCFT equations as

$$
\begin{gathered}
U_{J}(\boldsymbol{n})=\frac{\delta \mathcal{W}\left[\left\{\phi_{J}(\boldsymbol{n})\right\}\right]}{\delta \phi_{J}(\boldsymbol{n})}+u(\boldsymbol{n})=\sum_{K} \chi_{J K}\left\langle\left\langle\phi_{K}(\boldsymbol{n})\right\rangle\right\rangle+u(\boldsymbol{n}), \\
\phi_{J}(\boldsymbol{n})=\sum_{i} \sum_{N^{\prime}=1}^{N_{i}} \frac{n_{i} G_{i}\left(\boldsymbol{n}, N^{\prime}\right) G_{i}^{\dagger}\left(\boldsymbol{n}, N^{\prime}\right)}{\exp \left[-U_{f_{i}\left(N^{\prime}\right)}(\boldsymbol{n})\right] \sum_{\boldsymbol{n}} G_{i}\left(\boldsymbol{n}, N_{i}\right)} \delta\left[J, f_{i}\left(N^{\prime}\right)\right] .
\end{gathered}
$$

In eq. (5), $u(\boldsymbol{n})$ is a Lagrange multiplier which ensures the incompressibility condition. In the practical numerical procedure, we use the following recursive relations to calculate $G_{i}\left(\boldsymbol{n}, N^{\prime}\right)$ and $G_{i}^{\dagger}\left(\boldsymbol{n}, N^{\prime}\right)$ :

$$
\begin{aligned}
G_{i}\left(\boldsymbol{n}, N^{\prime}\right) & =\exp \left[-U_{f_{i}\left(N^{\prime}\right)}(\boldsymbol{n})\right]\left\langle\left\langle G_{i}\left(\boldsymbol{n}, N^{\prime}-1\right)\right\rangle\right\rangle \\
G_{i}^{\dagger}\left(\boldsymbol{n}, N^{\prime}\right) & =\exp \left[-U_{f_{i}\left(N^{\prime}\right)}(\boldsymbol{n})\right]\left\langle\left\langle G_{i}^{\dagger}\left(\boldsymbol{n}, N^{\prime}+1\right)\right\rangle\right\rangle,
\end{aligned}
$$

with the initial conditions $G_{i}(\boldsymbol{n}, 1)=\exp \left[-U_{f_{i}(1)}(\boldsymbol{n})\right]$ and $G_{i}^{\dagger}\left(\boldsymbol{n}, N_{i}\right)=\exp \left[-U_{f_{i}\left(N_{i}\right)}(\boldsymbol{n})\right]$.

We now extend the present lattice SCFT formulation in order to avoid the geometric constraints associated with a lattice. For this purpose, we redefine the ideal Hamiltonian in eq. (2) as [13]

$$
\begin{aligned}
\exp \left[-\hat{\mathcal{H}}_{0}(\Gamma)\right]= & \prod_{i, a, N^{\prime}}\left(\delta\left[\left|\boldsymbol{n}_{a, N^{\prime}+1}^{(i)}-\boldsymbol{n}_{a, N^{\prime}}^{(i)}\right|^{2}, 1\right]+\right. \\
& \left.+\frac{1}{2} \delta\left[\left|\boldsymbol{n}_{a, N^{\prime}+1}^{(i)}-\boldsymbol{n}_{a, N^{\prime}}^{(i)}\right|^{2}, 2\right]+\frac{1}{6} \delta\left[\left|\boldsymbol{n}_{a, N^{\prime}+1}^{(i)}-\boldsymbol{n}_{a, N^{\prime}}^{(i)}\right|^{2}, 3\right]\right) .
\end{aligned}
$$

This is an important extension in our work to deal with isotropic structures such as PME. According to eq. (9), the definition of the neighborhood average should be modified as

$$
\langle\langle X(\boldsymbol{n})\rangle\rangle=\sum_{\boldsymbol{n}^{\prime}}\left[\frac{6}{80} \delta\left[\left|\boldsymbol{n}-\boldsymbol{n}^{\prime}\right|^{2}, 1\right]+\frac{3}{80} \delta\left[\left|\boldsymbol{n}-\boldsymbol{n}^{\prime}\right|^{2}, 2\right]+\frac{1}{80} \delta\left[\left|\boldsymbol{n}-\boldsymbol{n}^{\prime}\right|^{2}, 3\right]\right] X\left(\boldsymbol{n}^{\prime}\right) .
$$

The above self-consistent set of equations are solved numerically by employing the iteration scheme used in refs. $[10,11,14]$. We first give an initial guess for $\left\{U_{J}(\boldsymbol{n})\right\}$ which can be either random or periodic in space. Then by using eqs. (7) and (8), the single-chain statistical weights $G_{i}\left(\boldsymbol{n}, N^{\prime}\right)$ and $G_{i}^{\dagger}\left(\boldsymbol{n}, N^{\prime}\right)$ are calculated, and the density fields $\left\{\phi_{J}(\boldsymbol{n})\right\}$ are evaluated by eq. (6). We finally update $\left\{U_{J}(\boldsymbol{n})\right\}$ according to both eq. (5) and the incompressibility condition. We repeat these steps until the variance of errors both in the multiplier $u(\boldsymbol{n})$ and in the local density $\sum_{J} \phi_{J}(\boldsymbol{n})$ becomes less than $10^{-8}$. Notice that the present algorithm is 
TABLE I - Characterization of the polymeric ternary systems. The parameter values in parentheses are for $1 \mathrm{D}$ systems whereas the others are for $2 \mathrm{D}$ systems (see the text).

\begin{tabular}{ccccccc}
\hline System & $\alpha$ & $\chi$ & $N$ & $N_{A B}$ & $\theta_{\mathrm{L}}$ & $(\chi N)_{\mathrm{L}}$ \\
\hline System 1 (S1) & 0.25 & $2(0.2)$ & $2(20)$ & $8(80)$ & $1 / 9$ & $9 / 4$ \\
System 2 (S2) & 0.5 & $2(0.2)$ & $4(40)$ & $8(80)$ & $1 / 3$ & 3 \\
System 3 (S3) & 1 & $2(0.2)$ & $8(80)$ & $8(80)$ & $2 / 3$ & 6 \\
System 4 (S4) & 2 & $2(0.2)$ & $16(160)$ & $8(80)$ & - & - \\
\hline
\end{tabular}

not intended to mimic the real polymer dynamics, but is an efficient artifice to evolve a system as fast as possible to a free energy minimum which can be either stable or metastable.

We consider symmetric ternary systems composed of equal-size $A$ and $B$ homopolymers and a symmetric $A B$ diblock copolymer. The common degree of polymerization of $A$ and $B$ homopolymers is denoted by $N$ and that of the block copolymer by $N_{A B}$. Such systems are characterized by two parameters, i.e., $\alpha=N / N_{A B}$ and $\chi N_{A B}\left(\chi=\chi_{A B}\right)$. We change the copolymer volume fraction $\theta$, keeping the volume fractions of the two homopolymers equal. Within the mean-field level, the isotropic Lifshitz point is located at $\theta_{\mathrm{L}}=2 \alpha^{2} /\left(1+2 \alpha^{2}\right)$ and $(\chi N)_{\mathrm{L}}=2\left(1+2 \alpha^{2}\right)[5]$. As listed in table I, we consider four different values of $\alpha$, but keeping $\chi N_{A B}=16$ which corresponds to the intermediate-segregation regime of pure diblock copolymers. We also fix the copolymer length, whereas the $A$ and $B$ homopolymer lengths are changed systematically. Notice that the Lifshitz point appears only for $\alpha \leq 1$ since it is pre-empted by the tricritical point for $\alpha>1$ [5].

First, we numerically solve the set of SCFT equations starting with randomly generated potential fields. This simulation is performed in 2 D systems $\left(L_{x}=L_{y}=256, L_{z}=1\right)$. Since the obtained patterns may depend on the initially prepared potential fields, we next study 1D systems starting with 1-dimensional periodic potential fields using the parameters written in the parentheses of table I. (We chose different values for 1D and 2D calculations in order to maximize the computational efficiency for large 2D systems.) The latter case yields lamellar structures with a well-defined periodicity.

Random initial conditions. - Let us denote the volume fractions of $A$ and $B$ homopolymers by $\phi_{A}$ and $\phi_{B}$, respectively, and that of $A B$ block copolymer by $\phi_{A B}$. Moreover, the volume fractions of $A$ and $B$ blocks in the copolymer is represented by $\phi_{a}$ and $\phi_{b}$, respectively. We then introduce three order parameters defined by $\Phi=\phi_{A}-\phi_{B}, \Psi=\phi_{a}-\phi_{b}$, and $\Delta=\phi_{A}+\phi_{a}-\phi_{B}-\phi_{b}$. Typical equilibrium patterns of $\Phi$ and $\Psi$ starting with randomly generated potential fields are shown in fig. 1 for S2 $(\alpha=0.5)$ and S4 $(\alpha=2)$. The patterns obtained for S1 and S3 are qualitatively similar to those of S2 although they are different from the patterns of S4. For $\theta=0.1$ of S2 most of the copolymers are located at the interface between $A$-rich and $B$-rich domains, and form saturated monolayers without any LRO. As $\theta$ is increased to 0.4 in S2 the amount of interface increases, but LRO still does not exist. Notice that this pattern which results from microphase separation resembles the experimentally observed PME structure [2]. For $\theta=0.9$ of S2, the system exhibits a lamellar structure (with defects) which is swollen by the small fraction of homopolymer chains. On the other hand, S4 $(\alpha=2)$ undergoes macrophase separation and we observe 3-phase coexistence between the copolymer-rich lamellar phase and the $A$-rich and $B$-rich homogeneous phases. This is clearly seen for $\theta=0.4$ of S4. Such a dependence on $\alpha$ is entirely consistent with the previous theoretical predictions in refs. $[5,8]$. 

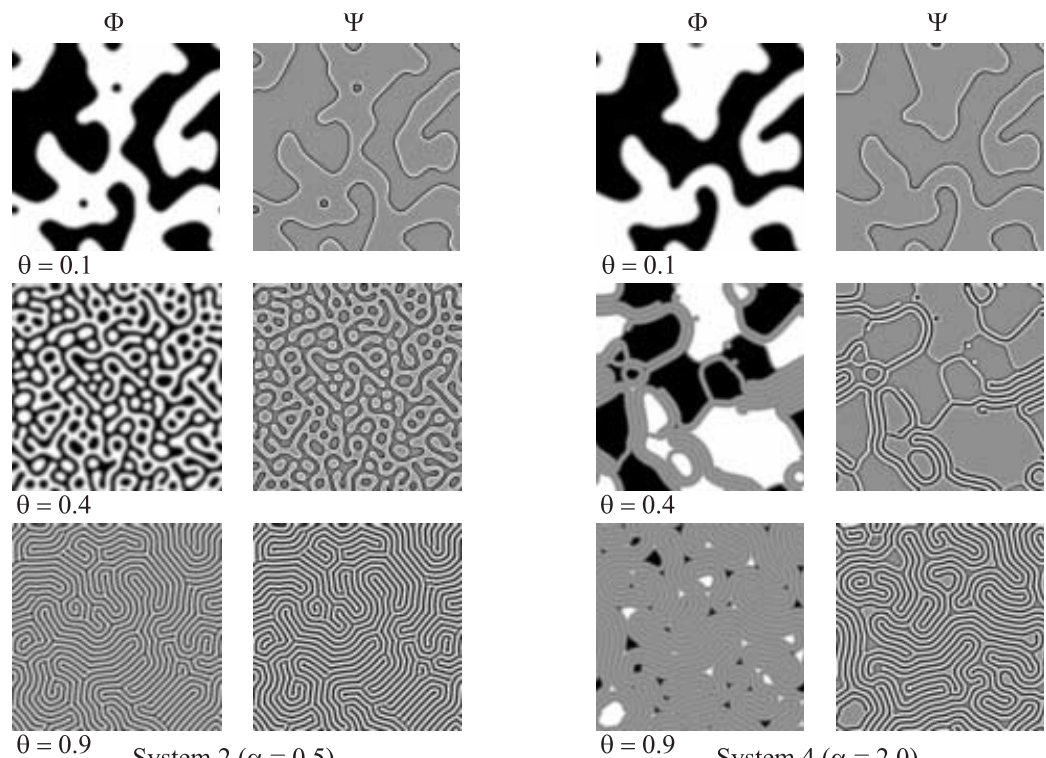

System $2(\alpha=0.5)$

System $4(\alpha=2.0)$

Fig. 1 - Typical 2D equilibrium patterns of System $2(\alpha=0.5)$ and System $4(\alpha=2.0)$ for three different values of $\theta . \Phi=\phi_{A}-\phi_{B}$ and $\Psi=\phi_{a}-\phi_{b}$. The dark area denotes the region of higher values.

For systems with $\alpha \leq 1$, we have calculated the following (inverse) characteristic length of each pattern:

$$
\langle k\rangle=\frac{\sum_{\boldsymbol{k} \neq \mathbf{0}}|\boldsymbol{k}|^{-1} S(\boldsymbol{k})}{\sum_{\boldsymbol{k} \neq \mathbf{0}}|\boldsymbol{k}|^{-2} S(\boldsymbol{k})} .
$$

Here $S(\boldsymbol{k})=\langle\Delta[\boldsymbol{k}] \Delta[-\boldsymbol{k}]\rangle$ is the structure function of all the $A$ and $B$ monomers, and $\Delta[\boldsymbol{k}]=$ $\sum_{\boldsymbol{k}} \Delta(\boldsymbol{k}) \exp [i \boldsymbol{k} \cdot \boldsymbol{n}]$ is the discrete Fourier transform of $\Delta(\boldsymbol{k})$ with $\boldsymbol{k}=2 \pi \boldsymbol{n} / L$ and $\boldsymbol{n} \in$ $\{0,1, \cdots, L-1\}^{2}$. In fig. $2,\langle k\rangle$ is plotted as a function of $\theta$ for different systems. We see that $\langle k\rangle$ is larger for relatively shorter homopolymer chain by which the penetration of the copolymer brushes becomes more efficient [15].

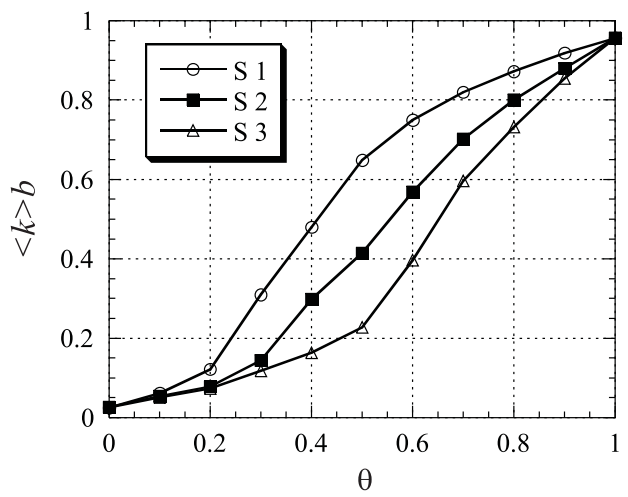

Fig. 2 - The inverse characteristic length $\langle k\rangle$ as a function of $\theta$ for different systems. 
Periodic initial conditions. - Since it is probable that the patterns in fig. 1 may correspond to metastable equilibrium, one should also pay attention to the existence of equilibrium phase with LRO. To check this point, we performed the same numerical calculations staring with the assumed 1-dimensional periodic potential fields and generated artificial lamellar structures. We then obtained the fully equilibrated lamellar phase by minimizing the free energy $F$ with respect to the lamellar spacing [16]. The resulting free energy $F$ is shown as a function of $\theta$ in fig. 3(a). In fig. 3(b), we plotted the deviation of the free energy from the tangent at $\theta=0.05$ for each curve in order to magnify its curvature. By carefully examining fig. 3(b), we find that there are three distinct regions for each curve; Region 1 (R1) where the profile is flat, Region $2(\mathrm{R} 2)$ where the curvature of the profile $\left(\partial^{2} F / \partial \theta^{2}\right)$ is negative, and Region 3 (R3) where the curvature is positive. These different regions are indicated in fig. 3 (b) for $\mathrm{S} 4$.

One should note that only R3 is locally stable. On the other hand, the flat part of the free energy in R1 indicates that the local stability of the lamellar phase is marginal. In this region, an arbitrary number of phases with different $\theta$ can coexist without any free energy cost. This means that the copolymer monolayers do not interact with each other, and any separation distance between the monolayers within the corresponding range is allowed. Similar behavior has been predicted for a highly swollen lamellar phase in binary blends [17]. We also find that $\phi_{A B}$ in the bulk region is constant $(\approx 0.01$ for all the systems in table $\mathrm{I})$ in $\mathrm{R} 1$. This constant value should coincide with the value of $\theta$ at which the lamellar periodicity diverges (unbinding transition) [8] and the free energy curve is smoothly connected to that of the 2-phase state. Since we are considering the intermediate-segregation regime $\left(\chi N_{A B}=16\right), \theta \approx 0.01$ is much smaller than the Lifshitz volume fraction $\theta_{\mathrm{L}}$. We also point out that $\mathrm{R} 1$ is larger for relatively shorter copolymer chain. This is consistent with the result in fig. 2 where the typical length scale $(\sim 1 /\langle k\rangle)$ is larger for larger $\alpha$.

Negative curvature in $\mathrm{R} 2$ results from the attractive interactions between the monolayers [17]. In this region, the lamellar phases in R1, R2 and a part of R3 are thermodynamically unstable, and macrophase separation into the lamellar, $A$-rich and $B$-rich phases (determined by a common tangent construction) should take place [5,8]. This unstable region hardly exists for S1 and S2, whereas it is remarkable for S4 and this is consistent with the 3 -phase coexistence shown in fig. 1 .
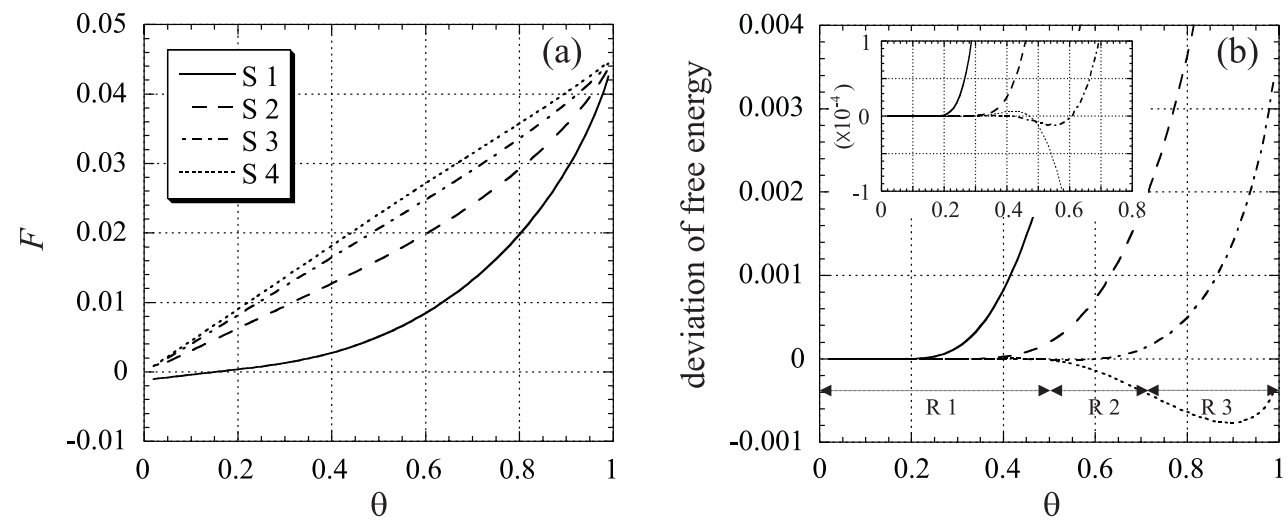

Fig. 3 - (a) The free energy $F$ as a function of $\theta$ for four different systems, and (b) its deviation from the tangent at $\theta=0.05$. The inset in (b) is the same plot magnified in the vertical axis. 
Discussion. - In order to discuss the stability of PME, one needs to know, for example, the entropic contribution due to the random configuration of interfaces, which is balanced with their bending energy [18]. Of course, this is beyond the scope of the present mean-field approach which showed the marginal stability of the strongly swollen lamellar phase. However, our result in fig. 1 with random initial conditions shows, at least in the static sense, that PME with random configuration of interfaces results from microphase separation as a metastable equilibrium. We comment that even the experimentally obtained PME may not necessarily be a fully equilibrated structure.

In summary, we have investigated the equilibrium structure of ternary blends of $A$ and $B$ homopolymers and symmetric $A B$ block copolymer by numerically solving the set of extended lattice SCFT equations. We showed that PME results from microphase separation for $\alpha \leq 1$ at least as a metastable equilibrium. Detailed free energy analysis revealed the marginal stability of the strongly swollen lamellar phase in the corresponding region. Further developments of the present study, such as 3D simulations, asymmetric ternary system, binary system, or different copolymer structure (e.g., $A B C$ triblock copolymer [19]) are in progress.

$$
* * *
$$

HK acknowledges support from the national project, which has been entrusted to the Japan Chemical Innovation Institute (JCII) by the New Energy and Industrial Technology Development Organization (NEDO) under MITI's Program for the Scientific Technology Development for Industries that Creates New Industries. We thank T. Kawakatsu, D. Andelman and H. ORLAnd for useful discussions.

\section{REFERENCES}

[1] Hamley I. W., The Physics of Block Copolymers (Oxford University Press, New York) 1998.

[2] Bates F. S. et al., Phys. Rev. Lett., 79 (1997) 849.

[3] Fredrickson G. H. and Bates F. S., J. Polym. Sci., Part B: Polym. Phys., 35 (1997) 2775.

[4] Leibler L., Macromolecules, 15 (1982) 1283.

[5] Broseta D. and Fredrickson G. H., J. Chem. Phys., 93 (1990) 2927.

[6] Holyst R. and Schick M., J. Chem. Phys., 96 (1992) 7728.

[7] Banaszak M. and Whitmore M. D., Macromolecules, 25 (1992) 249.

[8] Janert P. K. and Schick M., Macromolecules, 30 (1997) 137; 3916.

[9] Kielhorn L. and Muthukumar M., J. Chem. Phys., 107 (1997) 5588.

[10] Komura S., Kodama H. and Tamura K., in Slow Dynamics in Complex Systems, edited by M. TokuYama and I. Oppenheim (AIP, New York) 1999, pp. 188-189; Kodama H. and KomurA S., in Statistical Physics, edited by M. Tokuyama and H. E. Stanley (AIP, New York) 2000, pp. 247-249.

[11] Drolet F. and Fredrickson G. H., Phys. Rev. Lett., 83 (1999) 4317.

[12] Fleer G. J. et al., Polymers at Interfaces (Chapman \& Hall, London) 1993.

[13] This modification leads to a change of chain statistics. The resulting end-to-end distance of an ideal chain with length $N$ becomes $\left\langle R^{2}\right\rangle=1.65 b^{2} N$, whereas $\left\langle R^{2}\right\rangle=b^{2} N$ for the conventional lattice SCFT using eq. (2).

[14] Hasegawa R., Thesis, Nagoya University (1997).

[15] Dai K. H., Kramer E. J. and Shull K. R., Macromolecules, 25 (1992) 220.

[16] Noolandi J., Shi A.-C. and Linse P., Macromolecules, 29 (1996) 5907; Svensson M., Alexandridis P. and Linse P., Macromolecules, 32 (1999) 637.

[17] Matsen M. W., Phys. Rev. Lett., 74 (1995) 4225; Matsen M. W., Macromolecules, 28 (1995) 5765.

[18] Matsen M. W., J. Chem. Phys., 110 (1999) 4658.

[19] Fredrickson G. H. and Bates F. S., Eur. Phys. J., 1 (1998) 71. 